\title{
ACINIC CELL CARCINOMA OF PAROTID GLAND - A CASE REPORT
}

\section{Dr. J. Priyadharisini \\ Dr. Partho Protim Barman}

Assistant professor, Department of Pathology, Sri Lakshmi Narayana Institute of Medical Sciences, Osudu, Affliated to Bharath university

Professor, Department of Pathology, Sri Lakshmi Narayana Institute of Medical Sciences, Osudu, Affliated to Bharath university

Assistant professor, Department of Pathology, Sri Lakshmi Narayana Institute of Medical Sciences, Osudu, Affliated to Bharath university. ${ }^{*}$ Corresponding Author

ABSTRACT

Acinic cell carcinoma $(A C C)$ is a rare type of tumor which accounts for only $1-3 \%$ of the salivary gland tumors. Majority of which occurs in the parotid gland. ACC can be misinterpreted as benign because of its low grade histology. Radiological imaging techniques have low sensitivity in detecting malignancy of parotid glands. Guided FNAC can prove to be reliable diagnostic modality that can help in differential diagnosis of salivary gland neoplasms. Aspirates from these tumors can be diagnostically challenging. The lesions that have to be considered in the differential diagnosis include a wide variety of nonneoplastic as well as neoplastic conditions and pose a serious diagnostic dilemma cytologically. Hence histopathological examination is the gold standard method of choice for diagnosing these tumors. Here we report a case of Acinic cell carcinoma of parotid gland which had cytological diagnostic dilemma and confirmed diagnosis following histopathology examination.

\section{KEYWORDS :salivary Gland Tumor, Cytology Dilemma, Histopathology}

\section{INTRODUCTION}

The salivary gland acinic cell carcinoma $(\mathrm{ACC})$ is a rare malignant tumor. The acinic cell carcinoma (ACC) represents only $1-3 \%$ of the salivary gland tumors, most frequently affecting the parotid (1). Acinic cell carcinoma is the fourth most common malignant parotid gland tumour after mucoepidermoid carcinoma (MEC), adenoid cystic carcinoma and carcinoma ex pleomorphic adenoma A minor percentage found in the submandibular gland and minor salivary glands of the intraoral mucosa, palate, and upper lip $(2,3)$. Other locations of $\mathrm{ACC}$ in the human body could include the pancreas, stomach, prostate, breasts, lungs (4). Acinic cell carcinoma (ACC) occurs predominantly in females with a peak incidence in the third to fifth decade of life and seen frequently bilateral parotid region. It presents as a slowly enlarging mass in the parotid region with an indolent clinical course. This tumor has the tendency of local recurrence and metastatic spread to regional lymph nodes and distant sites. In general, ACCs are considered low-grade carcinomas and are treated conservatively. The majority is readily curable with surgical excision. A minority will recur or metastasize sometimes years after the initial diagnosis.

Although there is no reliable histopathologic feature that predicts an aggressive behavior, tumors with a predominant solid architecture, large size, lymphocytic infiltration, and cervical lymph node metastasis have shown a tendency to recur $(5-7)$. Despite the recognition of salivary gland fineneedle aspiration (FNA) as a sensitive and specific diagnostic tool (4-6), aspirates from ACC can present a diagnostic challenge because of their overlapping cytologic features with nonneoplastic conditions as well as with other benign and malignant tumors such as warthins tumor and mucoepidermoid carcinoma commonly (7), hence histopathological examination is the gold standard diagnostic method of choice .This article focuses on the cytomorphologic features that can help the pathologist arrive at an accurate cytologic diagnosis along with histopathological confirmation .

Case study

A 60-year-old female presented with progressively enlarging preauricular right cheek swelling for the last 1 year. She is known case of systemic hypertension for 10 years. On local examination, there was $5 \mathrm{~cm} \times 4 \mathrm{~cm}$ swelling over the right parotid region which was solid and immobile. Facial nerve function was normal. There was no swelling on the contralateral side. Ultrasonography suggested possible malignant salivary gland tumor.

Under aseptic precaution fine needle aspiration of right parotid swelling was done and smears were stained with Papanicolaou and giemsa, which revealed highly cellular smears showing cells arranged in clusters and single cells Some of these clusters showed loosely cohesive cells with overlapping nuclei and lack of polarity. Acinar formation was also observed. The cells are plump with near eccentric nuclei and had abundant, intracytoplasmic granular / vacuolated cytoplasm on the Papanicolaou-stained and giemsa preparations. The centrally located bland nuclei exhibited minimal pleomorphism. The background shows numerous lymphoid cells (Figure 1).

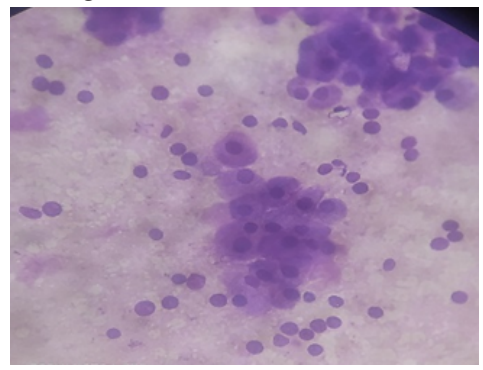

Figure.1-FNAC-40x- cellular smear with plump cells in loosely cohesive clusters and background of lymphoid cells

With these cytological features the following differential diagnosis were suggested which includes Acinic cell carcinoma, Warthin's tumor and low grade mucoepidermoid carcinoma.

Patient underwent total parotidectomy with prophylactic neck dissection with preservation of facial nerve. Lymph node dissection up to cervical level V was carried out. Surgical 
specimen sent for histopathology examination . Cut section revealed an ill defined grey white / brown tumor and 5 lymphnodes were identified. Sections from the tumor cells composed of predominantly acinar cells and scattered intercalated type cells which are arranged in solid sheets, nests and focal cystic areas. The tumor cells have vesicular nuclei in abundant finely granular basophilic cytoplasm resembling serous acinar cells. These cells are seen invading the surrounding fat. No atypical mitoses were identified. Lymphovascular invasion noted. $4 / 5$ of the lymph nodes showed evidence of tumor metastasis. Diagnosis of Acinic cell carcinoma was confirmed on histology (Figure2).

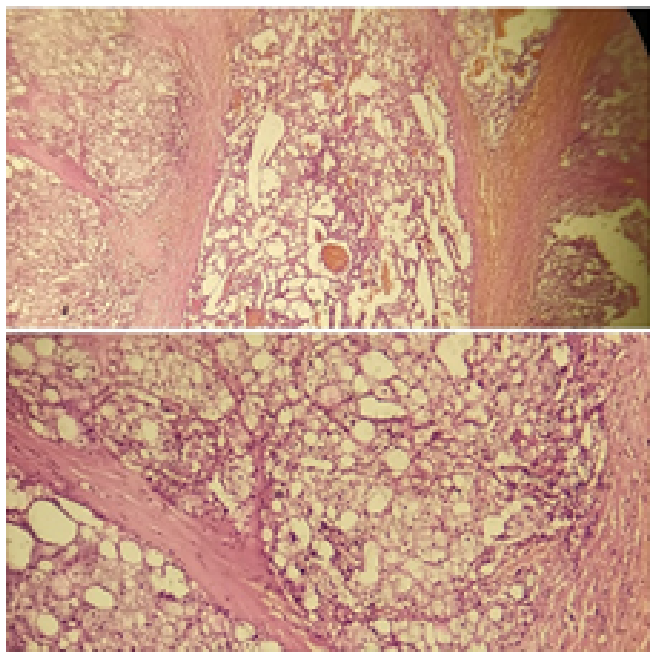

Figure.2-HPE- 40X-solid sheets and clusters of tumor cells showing abundant granular/vacuolated cytoplasm

\section{DISCUSSION}

Acinar cell carcinoma (ACC) is a salivary gland tumor that is characterized by proliferation of cells resembling serous acinar cells [8]. ACC is said to constitute $1-3 \%$ of all salivary gland tumors. Most commonly seen in females in the age group of 30-40 years . Radiation exposure is a predisposing factor for ACC . Parotid ACC typically presents with a slowly growing mass in the parotid region. Our patient also had history of gradually enlarging swelling over 1 year duration. Ultrasonography (USG) can be helpful for studying tumours topography and dimension and suggested possible malignant salivary gland tumor. Computed tomography (CT) and magnetic resonance imaging (MRI) are also useful providing additional information regarding the local extension $[9,10]$.

Fine-needle aspiration cytology (FNAC) is a rapid and safe diagnostic test in determining the nature of a parotid mass. When cellularity is sufficient FNAC has been reported to have a specificity of $91 \%$ and a sensitivity of $96 \%$ [11]. On cytology, ACC needs to be differentiated from two tumors, that is, mucoepidermoid carcinoma and warthin's tumor. Mucoepidermoid carcinoma shows dirty mucinous and necrotic background with the presence of malignant squamous, hybrid cells, and mucophages that are not seen in ACC (12). ACC showing abundant granular and dense cytoplasm of tumor cells with backgroung lymphocytes may be confused with warthin tumors. The presence of cytoplasmic metachromatic granules, the larger and more variable nuclei, and the numerous naked nuclei and lymphocytes found in the background of ACC can aid in the differentiation of these 2 neoplasms. In contrast to ACC, the cystic background in Warthin tumor is "dirty," with macrophages, cystic debris, and cholesterol clefts. The diagnosis was confirmed histopathologically. moderate consistency that can be solid or cystic. Usually, the tumor is less than $3 \mathrm{~cm}$ in diameter. The presented patient had a tumor with dimensions of $5 x 4 \mathrm{~cm}$, with grey white solid tumor on cut section. Microscopically, ACC is a tumor with multidirectional differentiation with various patterns: solid, micro-cystic, papillary, follicular, with or without hemorrhage. The tumor cells contain "zymogen granules", which are positive in the PAS staining. The mitotic figures are scarce(15). The differential diagnosis of the tumor in biopsy includes also mucoepidermoid carcinoma, pleomorphic adenoma, Warthin tumor, adenoid cystic carcinoma, sebaceous lymphadenoma, benign lymphoepithelial lesion, sialadenosis and radiation-induced sialadenitis. The new diagnostic entity, mammary analogue secretory carcinoma [20], should be also in the differential diagnosis of salivary gland tumors. However in our case had classic morphology with lymphnode showing metastasis.

\section{CONCLUSION}

Preoperative cytological diagnosis and associated imaging features can help surgeons to plan the extent of surgical treatment as complete surgical excision is the treatment of choice for most other malignant parotid tumors. Establishing a correct FNA diagnosis or the appropriate differential diagnosis can substantially alter the therapeutic management, preventing unnecessary extensive surgery.

\section{Financial support and sponsorship-Nil.}

\section{Conflicts of interest- There are no conflicts of interest.}

\section{REFERENCES}

1. Camellia Liana Buhas, Elena Rosca, Gabriela Mu Tiu, et al. Acinic cell carcinoma of minor salaivary gland - case report Rom J Morphol Embryol 2017;58(3): 1003-1007

2. Gnepp DR, Henley JD, Simpson R, Eveson J. Salivary and Lacrimal Glands. In: Diagnostic surgical pathology of the head and neck. Gnepp DR. Saunders 2nd edition 2009; 476-81

3. WM, Denneny JC 3rd, Atkins JP, et al. Acinic cell carcinoma of the oral cavity. Otolaryngol Head Neck Surg. 1982;90:696-699.

4. Kenner JR, Benson PM, Sinha C, et al. Acinic cell carcinoma presenting as an upper lip mass. Dermatol Surg. 1998;24:283-285.

5. Timon CI, Dardick I, Panzarella T, et al. Clinico-pathological predictors of recurrence for acinic cell carcinoma. Clin Otolaryngol. 1995;20:396-401.

6. Al-Khafaji BM, Nestok BR, Katz RL. Fine-needle aspiration of 154 parotid masses with histologic correlation: ten-year experience at the University of Texas M. D.Anderson Cancer Center. Cancer. 1998;84:153-159.

7. Klinjanienko J, Vielh P. Fine-needle sample of salivary gland lesions. V:cytology of 22 cases of acinic cell carcinoma with histologic correlation.Diagn Cytopathol. 1997; 17:347-352.

8. Tanaka K, Masuda M, Shinden S, et al. Fine-needle aspiration cytology of tumors of major salivary glands. Nippon Jibiinkoka Gakkai Kaiho.1998;101:1283-1291.

9. G. Seifert, Histological Typing of Salivary Gland Tumors, World Health Organization, Springer, 2nd edition, 1991.

10. Sato T, Kamata SE, Kawabata K, Nigauri T, Mitani H, Beppu T, et al. Acinic cell carcinoma of the parotid gland in a child. Pediatr Surg Int., 2005; 21(5): $377-80$

11. Levine SB, Potsic WP. Acinic cell carcinoma of the parotid gland in children. Int J Pediatr Otorhinolaryngol., 1986; 11 (3): 281-6.

12. Seema M., Syed Z.A. Fine Needle Aspiration: A Cytologic Study of Pediatric Lesions. Diagn Cytopathol., 1997; 17: 8-13.

13. Pinto A, Nosé V, Rojas C, Fan YS, Gomez-Fernandez C. Searching for mammary analog secretory carcinoma of salivary gland among its mimics. Mod Pathol., 2014; 27: 30-7

14. Coyne JD, Dervan PA. Primary acinic cell carcinoma of the breast. J Clin Pathol, 2002, 55(7):545-547.

15. Raymond MR, Yoo JH, Heathcote JG, et al. Accuracy of fine-needle aspiration biopsy for Warthin's tumours. J Otolaryngol. 2002;31:263-270. 